TAIWANESE JOURNAL OF MATHEMATICS

Vol. 12, No. 1, pp. 213-225, February 2008

This paper is available online at http://www.tjm.nsysu.edu.tw/

\title{
RAINBOW DOMINATION IN GRAPHS
}

\author{
Boštjan Brešar ${ }^{1}$, Michael A. Henning ${ }^{2}$ and Douglas F. Rall
}

\begin{abstract}
Assume we have a set of $k$ colors and to each vertex of a graph $G$ we assign an arbitrary subset of these colors. If we require that each vertex to which an empty set is assigned has in its neighborhood all $k$ colors, then this is called the $k$-rainbow dominating function of a graph $G$. The corresponding invariant $\gamma_{\mathrm{r} k}(G)$, which is the minimum sum of numbers of assigned colors over all vertices of $G$, is called the $k$-rainbow domination number of $G$. In this paper we connect this new concept to usual domination in (products of) graphs, and present its application to paired-domination of Cartesian products of graphs. Finally, we present a linear algorithm for determining a minimum 2-rainbow dominating set of a tree.
\end{abstract}

\section{INTRODUCTION}

Domination and its variations in graphs have been extensively studied, cf.[10,11]. For a graph $G=(V, E)$, a set $S$ is a dominating set if every vertex in $V \backslash S$ is adjacent to a vertex in $S$. The domination number $\gamma(G)$ is the minimum cardinality of a dominating set of $G$. We call a dominating set of cardinality $\gamma(G)$ a $\gamma(G)$-set. For subsets $S, T \subseteq V$, the set $S$ is said to dominate $T$ if every vertex of $T$ is adjacent to a vertex of $S$.

Domination represents situations in which each vertex/location that is not occupied by a guard needs to have a guard in a neighboring vertex/location. In these situations only one type of guards is considered. Assume a more complex situation where there are different types of guards (let there be $k$ such types), and we require that each vertex/location that is not occupied with a guard has in its neighborhood all types of guards. This relaxation leads to the following definition.

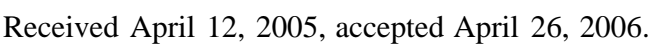

Communicated by Xuding Zhu.

2000 Mathematics Subject Classification: 05C69.

Key words and phrases: Domination, Paired-domination, Cartesian product, Vizing's conjecture.

${ }^{1}$ Supported by the Ministry of Science of Slovenia under the grant P1-0297. ${ }^{2}$ Research supported in part by the South African National Research Foundation and the University of KwaZulu-Natal. 
Let $G$ be a graph and let $f$ be a function that assigns to each vertex a set of colors chosen from the set $\{1, \ldots, k\}$; that is, $f: V(G) \rightarrow \mathcal{P}(\{1, \ldots, k\})$. If for each vertex $v \in V(G)$ such that $f(v)=\emptyset$ we have

$$
\bigcup_{u \in N[v]} f(u)=\{1, \ldots, k\}
$$

then $f$ is called the $k$-rainbow dominating function (kRDF) of $G$. The weight, $w(f)$, of a function $f$ is defined as $w(f)=\sum_{v \in V(G)}|f(v)|$. Given a graph $G$, the minimum weight of a kRDF is called the k-rainbow domination number of $G$, which we denote by $\gamma_{\mathrm{r} k}(G)$.

Another variation of domination that we consider in this paper is already wellknown. It is called paired-domination and was introduced by Haynes and Slater in $[12,13]$ as a model for assigning backups to guards for security purposes. It is studied, for example, in [7, 14-16] and elsewhere. A matching in a graph $G$ is a set of independent edges in $G$. A perfect matching $M$ in $G$ is a matching such that every vertex of $G$ is incident to an edge of $M$. A paired-dominating set, denoted PDS, of a graph $G$ is a set $S$ of vertices of $G$ such that every vertex is adjacent to some vertex in $S$ and the subgraph $G[S]$ induced by $S$ contains a perfect matching $M$ (not necessarily induced). Two vertices joined by an edge of $M$ are said to be paired in $S$ and are called partners (with respect to $M$ ). Every graph without isolated vertices has a PDS since the end-vertices of any maximal matching form such a set. The paired-domination number of $G$, denoted by $\gamma_{\mathrm{pr}}(G)$, is the minimum cardinality of a PDS. A PDS of cardinality $\gamma_{\mathrm{pr}}(G)$ we call a $\gamma_{\mathrm{pr}}(G)$-set.

In general we follow the notation and graph theory terminology in $[6,10]$. Specifically, let $G=(V, E)$ be a graph with vertex set $V$ and edge set $E$. For any vertex $v \in V$, the open neighborhood of $v$ is the set $N(v)=\{u \in V \mid u v \in E\}$, and its closed neighborhood is the set $N[v]=N(v) \cup\{v\}$. If $A \subset V$ then $N[A]$ denotes the union of closed neighborhoods of vertices of $A$. A vertex of degree one is called a leaf and its neighbor is called a support vertex. For graphs $G$ and $H$, the Cartesian product $G \square H$ is the graph with vertex set $V(G) \times V(H)$ where two vertices $\left(u_{1}, v_{1}\right)$ and $\left(u_{2}, v_{2}\right)$ are adjacent if and only if either $u_{1}=u_{2}$ and $v_{1} v_{2} \in E(H)$ or $v_{1}=v_{2}$ and $u_{1} u_{2} \in E(G)$.

Rainbow domination in a graph $G$ has a natural connection with the study of $\gamma\left(G \square K_{k}\right)$. If the vertex set of $K_{k}$ is $\left\{x_{1}, \ldots, x_{k}\right\}$, then there is a one-to-one correspondence between the set of kRDFs of $G$ and the dominating sets of $G \square K_{k}$. For a given $\operatorname{kRDF} f$, of $G$, the set

$$
D_{f}=\bigcup_{v \in V(G)}\left(\bigcup_{i \in f(v)}\left\{\left(v, x_{i}\right)\right\}\right),
$$

is a dominating set of $G \square K_{k}$. The reverse correspondence is clear. This proves the following result. 
Observation 1.1. For $k \geq 1$ and for every graph $G, \gamma_{\mathrm{r} k}(G)=\gamma\left(G \square K_{k}\right)$.

This connection was explored further-in the language of domination of Cartesian products-by Hartnell and Rall for the special case of $k=2$. In [9] they derive many properties of graphs $G$ with $\gamma_{\mathrm{r} 2}(G)=\gamma(G)$. In particular, it follows that for any tree $T, \gamma(T)<\gamma_{\mathrm{r} 2}(T)$. In addition they observed that no graph has $\gamma=\gamma_{\mathrm{r} k}$ for $k \geq 3$. Expressed in terms of rainbow domination their Theorem 5 yields the following sharp bounds.

Observation 1.2. [9] Let $G$ be a graph. Then for any $k \geq 2$,

$$
\min \{|G|, \gamma(G)+k-2\} \leq \gamma_{\mathrm{r} k}(G) \leq k \gamma(G) .
$$

The attempt in [9] to characterize graphs with $\gamma=\gamma_{\mathrm{r} 2}$ was inspired by the following famous open problem.

Vizing's Conjecture. For any graphs $G$ and $H, \gamma(G \square H) \geq \gamma(G) \gamma(H)$.

The conjecture motivated several authors to consider different kinds of domination problems in Cartesian products. See the survey [8], and some more recent results on the topic $[1,5,15,17]$. One of the related problems posed in [8] is to find classes of graphs that achieve the equality. There it was shown that $\gamma(G \square H)=\gamma(G) \gamma(H)$, if $G$ is any graph with $\gamma(G)=\gamma_{\mathrm{r} 2}(G)$ and $H$ is a socalled generalized comb.

The introduction of rainbow domination was motivated by the study of paireddomination in Cartesian products of graphs, where certain upper bounds can be expressed in terms of rainbow domination. We present these applications in the next section. In the last section we present a linear algorithm for determining a $\gamma_{\mathrm{r} 2}$-set of a tree.

\section{Paired-domination of Cartesian Products}

Our aim in this section is to determine an upper bound for the paired-domination number of Cartesian products of graphs using rainbow domination in graphs. We will use this bound in determining the exact values of the paired-domination number for some products of cycles.

\subsection{Preliminary observations}

In this section, we present some preliminary results on the paired-domination number.

We start with a trivial upper bound on $\gamma_{\mathrm{pr}}(G \square H)$. First note that it is not true that $\gamma_{\mathrm{pr}}(G \square H) \leq \gamma_{\mathrm{pr}}(G) \gamma_{\mathrm{pr}}(H)$ in general. For example, if $n>4$, then $\gamma_{\mathrm{pr}}\left(K_{n} \square K_{n}\right) \geq n$ and $\gamma_{\mathrm{pr}}\left(K_{n}\right)=2$. We do however have the following 
Observation 2.1. For any graphs $G$ and $H$ without isolated vertices,

$$
\gamma_{\mathrm{pr}}(G \square H) \leq \min \left\{\gamma_{\mathrm{pr}}(G)|V(H)|, \gamma_{\mathrm{pr}}(H)|V(G)|\right\} .
$$

Proof. Let $\left\{u_{1}, v_{1}, \ldots, u_{k}, v_{k}\right\}$ be a $\gamma_{\mathrm{pr}}(G)$-set with $u_{i}$ and $v_{i}$ paired (and so $\left.\gamma_{\mathrm{pr}}(G)=2 k\right)$, and let $V(H)=\{1,2, \ldots, h\}$. Then the set of all vertices of the form $\left(u_{i}, j\right)$ and $\left(v_{i}, j\right)$ where $1 \leq i \leq k$ and $1 \leq j \leq h$ is a PDS of $G \square H$ (with $\left(u_{i}, j\right)$ and $\left(v_{i}, j\right)$ paired), and so $\gamma_{\mathrm{pr}}(G \square H) \leq 2 k h=\gamma_{\mathrm{pr}}(G)|V(H)|$.

Observation 2.1. For any graph $G=(V, E)$ without isolated vertices of order $n$ and maximum degree $\Delta$,

$$
\gamma_{\mathrm{pr}}(G) \geq 2\left\lceil\frac{n}{2 \Delta}\right\rceil
$$

Proof. Let $S$ be a $\gamma_{\mathrm{pr}}(G)$-set. Then, $n-|S|=|V \backslash S|=\mid \cup_{v \in S}(N(v) \cap(V \backslash$ $S))|\leq| S \mid \cdot(\Delta-1)$, and so $\gamma_{\mathrm{pr}}(G)=|S| \geq n / \Delta$. The desired lower bound now follows since $\gamma_{\mathrm{pr}}(G)$ is even.

As an immediate consequence of Observation 2.2, we have another lower bound on the paired-domination number of the Cartesian product of two graphs.

Observation 2.3. For any pair of graphs $G$ and $H$,

$$
\gamma_{\mathrm{pr}}(G \square H) \geq 2\left\lceil\frac{|V(G)||V(H)|}{2(\Delta(G)+\Delta(H))}\right\rceil .
$$

\subsection{Bounds using rainbow domination}

Theorem 2.4. For any graph $G$ and any graph $H$ whose vertex set can be partitioned into $k \gamma_{\mathrm{pr}}(H)$-sets,

$$
\gamma_{\mathrm{pr}}(G \square H) \leq \frac{1}{k}|V(H)| \gamma_{\mathrm{r} k}(G) .
$$

Proof. Let $f$ be a kRDF of $G$ of minimum weight, and let $S_{1}, S_{2}, \ldots, S_{k}$ form the partition of $V(H)$ into $k \gamma_{\mathrm{pr}}(H)$-sets. Note that $\gamma_{\mathrm{pr}}(H)=\left|S_{i}\right|=\frac{1}{k}|V(H)|$ for all $i \in\{1, \ldots, k\}$.

For each $v \in V(G)$, we define a subset $D_{v} \subseteq\{v\} \times V(H)$ as

$$
D_{v}=\bigcup_{i \in f(v)}\left(\{v\} \times S_{i}\right) .
$$


That is, if $f(v)=\emptyset$, then $D_{v}$ is also empty; otherwise $D_{v}$ consists of sets corresponding to $S_{i}$ where $i$ runs through $f(v)$. Let

$$
D=\bigcup_{v \in V(G)} D_{v}
$$

We show that $D$ is a PDS of $G \square H$. Let $v \in V(G)$. If $f(v) \neq \emptyset$ then $D_{v}$ contains at least one set $\{v\} \times S_{i}$ which already dominates $\{v\} \times V(H)$. Suppose $f(v)=\emptyset$, and let $(v, x)$ be arbitrary vertex of $\{v\} \times V(H)$, where $x \in S_{j}$ for some $j$. By definition of $k$-rainbow domination

$$
\bigcup_{u \in N[v]} f(u)=\{1, \ldots, k\},
$$

hence there exist a vertex $u \in N_{G}(v)$ such that $j \in f(u)$, and so $(u, x) \in D_{u} \subset D$. Thus $(v, x)$ is dominated by $(u, x) \in D$, and we conclude that $D$ is a dominating set. It follows from the definition of $D$ that $G[D]$ has a perfect matching, and hence $D$ is a PDS.

Clearly

$$
\begin{aligned}
|D| & =\sum_{v \in V(G)}\left|D_{v}\right|=\sum_{v \in V(G)}|f(v)| \cdot \gamma_{\mathrm{pr}}(H) \\
& =\frac{1}{k}|V(H)| \sum_{v \in V(G)}|f(v)|=\frac{1}{k}|V(H)| \gamma_{\mathrm{r} k}(G),
\end{aligned}
$$

and the proof is complete.

Combining Theorem 2.4 with Observation 1.1, we get the following result.

Corollary 2.5. For any graph $G, \gamma_{\mathrm{pr}}\left(G \square\left(K_{k} \square K_{2}\right)\right) \leq 2 \gamma_{\mathrm{r} k}(G)=2 \gamma\left(G \square K_{k}\right)$. In particular, $\gamma_{\mathrm{pr}}\left(G \square C_{4}\right) \leq 2 \gamma_{\mathrm{r} 2}(G)$.

Let us now concentrate on the paired-domination number of products of cycles with the 4-cycle. First we determine an upper bound on the 2-rainbow domination number of a cycle.

Observation 2.6. For $n \geq 3, \gamma_{r 2}\left(C_{n}\right) \leq\lfloor n / 2\rfloor+\lceil n / 4\rceil-\lfloor n / 4\rfloor$.

Proof. Let $C_{n}$ be the cycle $v_{1}, v_{2}, \ldots, v_{n}, v_{1}$. Let $g: V\left(C_{n}\right) \rightarrow \mathcal{P}(\{1,2\})$ be defined as follows: Let $g\left(v_{i}\right)=\{1\}$ if $i \equiv 1(\bmod 4), g\left(v_{i}\right)=\{2\}$ if $i \equiv 3(\bmod 4)$, and $g\left(v_{i}\right)=\emptyset$ otherwise. If $n \not \equiv 2(\bmod 4)$, let $f=g$, while if $n \equiv 2(\bmod 4)$, let $f\left(v_{i}\right)=g\left(v_{i}\right)$ for $1 \leq i \leq n-1$ and let $f\left(v_{n}\right)=\{2\}$. Then, $f$ is a $2 \mathrm{RDF}$ of $C_{n}$. If $n \not \equiv 2(\bmod 4)$, then the weight of $f$ is $w(f)=\lceil n / 2\rceil=\lfloor n / 2\rfloor+\lceil n / 4\rceil-\lfloor n / 4\rfloor$, 
while if $n \equiv 2(\bmod 4), w(f)=(n+2) / 2=\lfloor n / 2\rfloor+\lceil n / 4\rceil-\lfloor n / 4\rfloor$. The desired result follows since $\gamma_{r 2}\left(C_{n}\right) \leq w(f)$.

Proposition 2.7. For $n \geq 3, \gamma_{\mathrm{pr}}\left(C_{n} \square C_{4}\right)=2 \gamma_{r 2}\left(C_{n}\right)$. That is,

$$
\gamma_{\mathrm{pr}}\left(C_{n} \square C_{4}\right)= \begin{cases}2\lceil n / 2\rceil & \text { if } n \not \equiv 2(\bmod 4) \\ n+2 & \text { otherwise. }\end{cases}
$$

Proof. Observation 2.3 implies $\gamma_{\mathrm{pr}}\left(C_{n} \square C_{4}\right) \geq 2\lceil n / 2\rceil$. Hence as a consequence of Observation 2.3, Corollary 2.5 and Observation 2.6, we derive in the case $n \not \equiv 2(\bmod 4)$ that $\gamma_{\mathrm{pr}}\left(C_{n} \square C_{4}\right)=2\lceil n / 2\rceil$. Now let $n \equiv 2(\bmod 4)$. The same arguments imply that $\gamma_{\mathrm{pr}}\left(C_{n} \square C_{4}\right)$ is equal to $n$ or $n+2$. Suppose $\gamma_{\mathrm{pr}}\left(C_{n} \square C_{4}\right)=n$ and let $D$ be a minimum PDS of $C_{n} \square C_{4}$. Observe that two adjacent vertices of $D$ dominate exactly 8 vertices. This can be achieved only if every vertex of $C_{n} \square C_{4}$ is adjacent to exactly one vertex of $D$. It is an easy exercise to check that this is impossible, and hence $\gamma_{\mathrm{pr}}\left(C_{n} \square C_{4}\right)=n+2$ in this case.

\section{2-Rainbow Domination IN TREes}

It is well-known that a dominating set of a tree can be found in linear time and space [4]. In this section we present a linear algorithm for determining a $\gamma_{\mathrm{r} 2}$-set of an arbitrary tree.

We begin with the following easy observation. If $T$ is a path, then the following $2 \mathrm{RDF}$ is minimum. Start by labelling a leaf by one symbol (say 1), then label each second vertex by one symbol in an alternating way (so the third vertex gets 2 , then fifth again 1 , and so on), until we reach the other leaf or its neighbor, and in the latter case just add a symbol also to this other leaf. (We get $\gamma_{\mathrm{r} 2}\left(P_{n}\right)=\lfloor n / 2\rfloor+1$.) Our algorithm for 2-rainbow domination of general trees evolves from the above algorithm for paths. (In fact, if $T$ is a path, and for the root we choose a leaf, our algorithm performs the described procedure).

Our aim in this section is to present a linear algorithm for determining a $\gamma_{\mathrm{r} 2}(T)$ set of an arbitrary tree $T$. For this purpose, we introduce another type of domination, which could also be called a monochromatic version of rainbow domination. In general it is a simplification of the rainbow domination, yet in trees both variations are equivalent.

Let $G=(V, E)$ be a graph and let $f$ be a function that assigns to each vertex a number chosen from $\{0,1,2\}$ called its weight; that is, $f: V \rightarrow\{0,1,2\}$. For $v \in V$, we define

$$
f[v]=\sum_{u \in N[v]} f(u)
$$


for notational convenience. We call a vertex $v \in V$ a bad vertex with respect to $f$ if $f(v)=0$ and $f[v] \leq 1$; otherwise, we say that $v$ is a good vertex with respect to $f$. Note that if $v$ is a good vertex with respect to $f$ and $f(v)=0$, then $f[v] \geq 2$. If every vertex of $T$ is a good vertex with respect to $f$, then $f$ is called a weak $\{2\}$-dominating function (W2DF) of $G$. The weight $w(f)$ of $f$ is defined as $w(f)=\sum_{v \in V} f(v)$. The minimum weight of a W2DF in $G$ is called the weak $\{2\}$-domination number of $G$, which we denote by $\gamma_{w 2}(G)$. A W2DF in $G$ of weight $\gamma_{w 2}(G)$ we call a $\gamma_{w 2}(G)$-function.

Observation 3.1. For every tree $T, \gamma_{\mathrm{r} 2}(T)=\gamma_{w 2}(T)$.

Proof. Let $T=(V, E)$ and let $g$ be a $2 \mathrm{RDF}$ of $T$ of minimum weight, i.e., let $g$ be a $\gamma_{\mathrm{r} 2}(T)$-function. Let $f_{g}: V \rightarrow\{0,1,2\}$ be defined by $f_{g}(v)=|g(v)|$ for all $v \in V$. Then, $f_{g}$ is a W2DF of $T$ of weight $w\left(f_{g}\right)=w(g)=\gamma_{\mathrm{r} 2}(T)$, and so $\gamma_{w 2}(T) \leq w\left(f_{g}\right)=\gamma_{\mathrm{r} 2}(T)$.

It remains for us to show that $\gamma_{w 2}(T) \geq \gamma_{\mathrm{r} 2}(T)$. Let $f$ be a $\gamma_{w 2}(T)$-function. Let $g_{f}: V \rightarrow \mathcal{P}(\{1,2\})$ be defined as follows: If $f(v)=0$, let $g_{f}(v)=\emptyset$. If $f(v)=2$, let $g_{f}(v)=\{1,2\}$. If $f(v)=1$, let $g_{f}(v)$ be chosen so that (i) $g_{f}(v)=\{1\}$ or $g_{f}(v)=\{2\}$ and (ii) the number of vertices $v$ for which $g_{f}(v) \neq \emptyset$ or $\bigcup_{u \in N[v]} g_{f}(u)=\{1,2\}$ is a maximum (loosely speaking the condition (ii) states that the number of vertices that are dominated by $g_{f}$ in the sense of 2-rainbow domination is a maximum). We show that then for every vertex $v \in V(G)$ we have $g_{f}(v) \neq \emptyset$ or $\bigcup_{u \in N[v]} g_{f}(u)=\{1,2\}$ (that is $g_{f}$ is a $2 \mathrm{RDF}$ of $T$ ). Assume, to the contrary that there is vertex $v$ not having this property with respect to $g_{f}$. Since $v$ is good with respect to $f$, we infer that $f(v)=0$, no neighbor of $v$ has weight 2 under $f$, all neighbors of $v$ have weight at most 1 under $f$, and at least two of them, say $x$ and $y$, have weight exactly 1 . We may assume without loss of generality that every neighbor $z$ of $v$ with $f(z)=1$ satisfies $g_{f}(z)=\{1\}$. Let $T_{x}$ be the component of $T-v$ containing $x$. Perform a bichromatic exchange in the subtree $T_{x}$ (that is, change all 1's to 2 's and vice-versa), and let $g_{f}^{\prime}: V \rightarrow \mathcal{P}(\{1,2\})$ denote the resulting function. Every vertex $z$ in $T_{x}$ preserves the desired property, if it holds under $g_{f}$, while $\bigcup_{u \in N[v]} g_{f}^{\prime}(u)=\{1,2\}$ since $g_{f}^{\prime}(x)=\{2\}$ and $g_{f}^{\prime}(y)=\{1\}$. This is contrary to our choice of $g_{f}$. We deduce, therefore, that $g_{f}$ is a $2 \mathrm{RDF}$ of $T$, and so $\gamma_{\mathrm{r} 2}(T) \leq w\left(g_{f}\right)=w(f)=\gamma_{w 2}(T)$.

Note that the above observation need not be true if a graph has cycles. For instance $\gamma_{\mathrm{r} 2}\left(C_{6}\right)=4>3=\gamma_{w 2}\left(C_{6}\right)$.

Once a minimum W2DF of a tree is obtained it is easy to determine a $2 \mathrm{RDF}$ of $T$ with the same weight, and one can perform this in linear time. So, henceforth we shall consider the problem of determining a minimum W2DF of a tree.

In the algorithm we will use the following sets: 
$O$ - vertices that have not yet been checked by the algorithm,

$D$ - vertices with assigned positive weight, that partitions into

$D_{1}$ - vertices with assigned 1 , and $D_{2}$ - vertices with assigned 2,

$N_{1}$ - vertices not in $D$ whose sum of weights in its neighborhood is 1 ,

$N_{2}$ - vertices not in $D$ whose sum of weights in its neighborhood is at least 2,

$L$ - a dynamic set of leaves (updated after each deletion of a vertex).

The goal is that the sets $D_{1}$ and $D_{2}$ induce a W2DF (obtained by assigning 2 to vertices of $D_{2}, 1$ to vertices of $D_{1}$ and 0 to all other vertices of $T$ ). Clearly, at the end of the algorithm all vertices should be either in $D$ or $N_{2}$, with $2\left|D_{2}\right|+\left|D_{1}\right|$ minimized at the same time.

We will use a common data structure called "parent array". By choosing a root $r$ arbitrarily, the function $P(v)$ yields the parent of $v$ with respect to $r$ (that is the neighbor of $v$ in the path to $r$ ). In each step of the algorithm we choose a leaf $v$, update his and his parent's location with respect to the above sets, and then delete $v$ from $T$ (which also includes the decrease of degree of $P(v)$ and updating $L$ ). In the beginning $L$ consists of leaves of $T$. After each step (deletion of $v$ from $T$ ) $v$ is deleted from $L$, and if $P(v)$ obtains degree 1 , it is added to $L$. The only exception is $r$ which is never added to $L$.

In the proof of the correctness of the algorithm we shall also use the following notation. Given a vertex $v$ by $B(v)$ we denote the subtree of $T$, consisting of $v$ and all descendants of $v$ with respect to $r$ (vertices that are "below" $v$ ) We call this subtree the below subtree of $v$ with respect to $r$. Note that when vertex $v$ is visited, all vertices of $B(v)-v$ have already been deleted from $T$.

By a proleaf we mean a vertex $v$ that is either a leaf of $T$ or when $v$ is visited (during the the algorithm) all the neighbors of $v$ in $B(v)$ are in $N_{2}$ (so they do not need its weight for their domination, and they do not pass any weight to $v$ ).

In the course of the algorithm two special rules are applied which carry the main idea. The rules instruct when the vertices are added to $D_{2}$. Let $v$ be a vertex visited by the algorithm.

Rule 1. If $v$ has been assigned weight 1 during the algorithm (because of requirements of its descendants) and $v$ is adjacent to at least one proleaf then reassign to $v$ weight 2 , and assign 0 to all its proleaves.

Rule 2. If $v$ has been assigned weight 0 during the algorithm, and $v$ is adjacent to at least two proleafs then reassign to $v$ weight 2 , and assign 0 to all its proleaves.

Operation set $v \in S$ at the same time removes $v$ from a set to which it belongs and puts it in $S$. The boolean function must $(v)$, if true for a vertex $v$ means that when $v$ is visited, it must be put in $D_{1}$ (because of requirement of its descentant). In 
the beginning $\operatorname{must}(v)=$ false for all $v \in T$. It becomes true for the parent of a vertex $v$ which is in $N_{1}$. Function $\operatorname{supp}(v)$ gives a list of vertices that are supported by $v$, more precisely, they are the proleaves adjacent to $v$. In the beginning $\operatorname{supp}(v)=\emptyset$ for all $v \in T$. The list is needed in relation with the condition of the two rules.

\section{Algorithm 2-Rainbow domination of a tree}

Input: a tree $T$, its root $r$, the list of leaves $L$

Output: sets $D_{1}$ and $D_{2}$ at the end of the algorithm induce a $\gamma_{w 2}(\mathrm{~T})$-function.

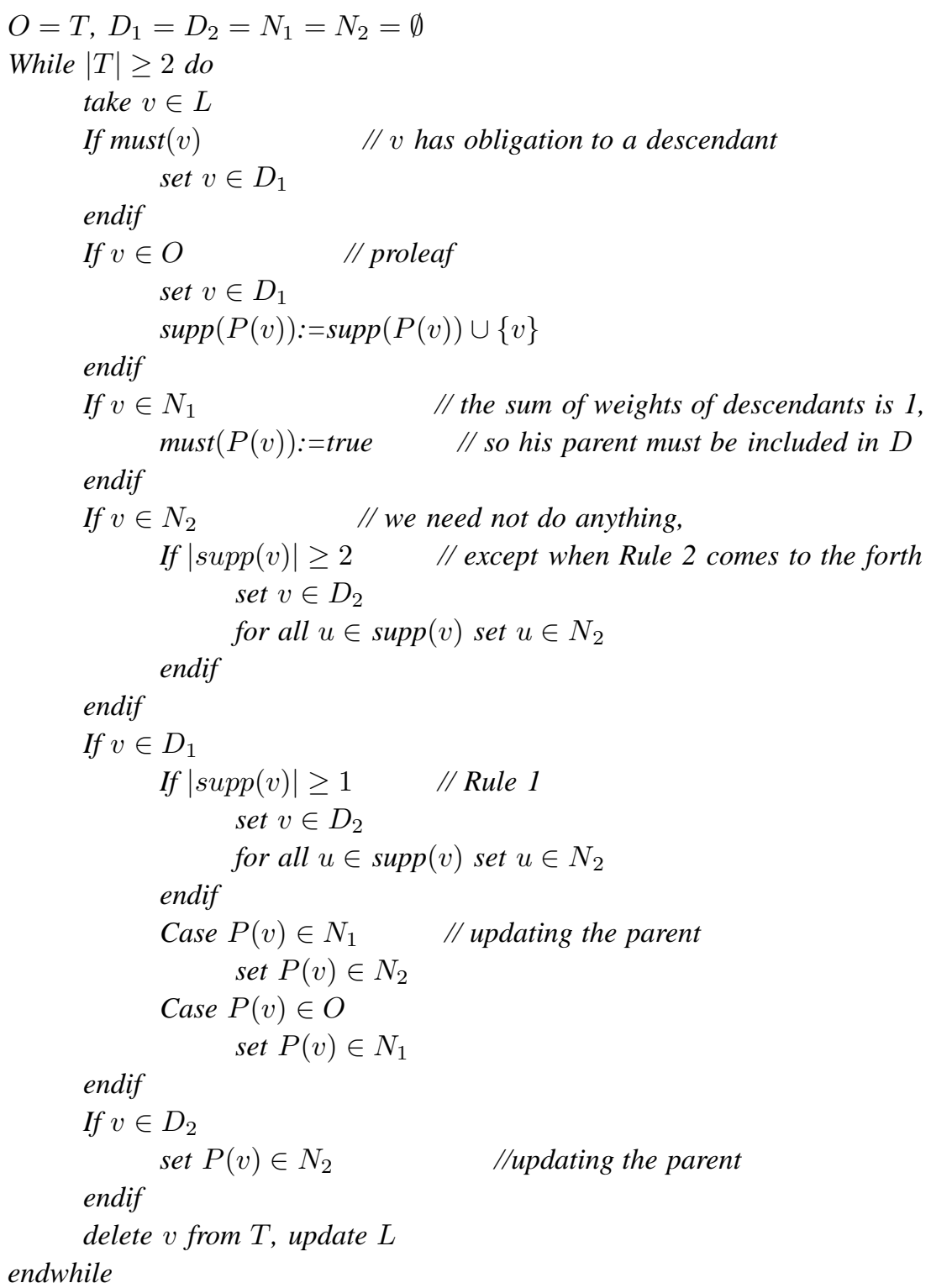




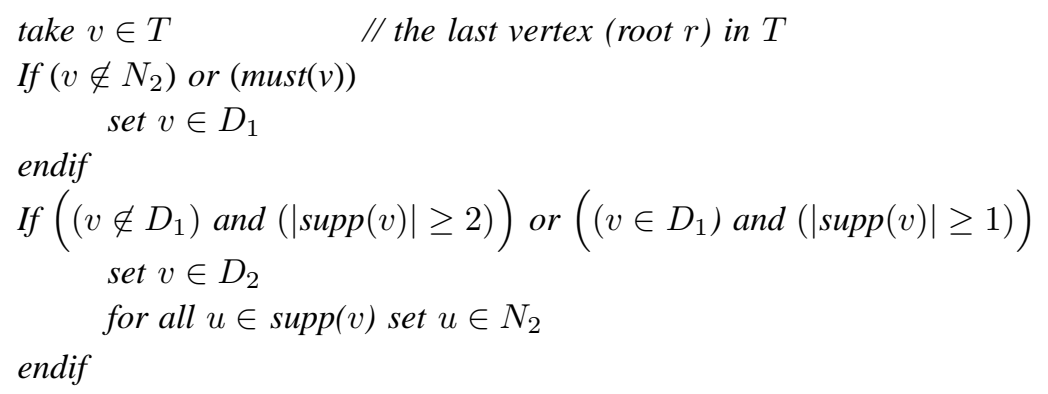

Theorem 3.2. Algorithm 2-Rainbow domination of a tree gives a W2DF of minimum weight. Its time and space complexity is $O(n)$ where $n$ is the number of vertices of the tree.

Proof. The complexity part of the theorem is easy since in each turn of the While sentence only a constant number of operations are performed, and there are $n-1$ such turns (for all vertices except $r$ ). The last two If sentences are performed in constant time. For the correctness of the algorithm we will use the following statement.

Claim. Let $v$ be a vertex added to $D\left(D_{1}\right.$ or $\left.D_{2}\right)$ in the course of the algorithm, Then $D_{1} \cap B(v)$ and $D_{2} \cap B(v)$ induce a minimum weight W2DF for the below subtree $B(v)$ of $v$, such that, in addition, $v$ has as big weight as possible.

Proof of Claim. The proof is by induction on the size of the below subtree $B(v)$. If $B(v)=\{v\}$ then $v$ must be a leaf of $T$, and so it is put in $D_{1}$ by the algorithm. Clearly this is an optimal W2DF of $B(v)$.

Let $|B(v)|>1$, and suppose $v$ is put in $D$ by the algorithm. Note that some vertices are already in $D \cap B(v)$. We will check all possible places in the algorithm in which $v$ is added to $D$, and prove that the claim holds in every such case.

Suppose we add $v$ to $D_{1}$ in the first If sentence (because of $m u s t(v)=$ true). This means that there exists a child $u$ of $v$ such that $u \in N_{1}$. Hence $u$ has only one child $x$ that is in $D$, more precisely $x \in D_{1}$. Since $B(x) \cap D$ is optimal in $B(x)$ with weight of $x$ as large as possible, we derive that also $B(v) \cap D$ is optimal when we add $v$ to $D_{1}$ (regardless if $v$ has other children except $u$ ).

Suppose $v \in O$ and $v$ is not a leaf (the case of leaves was cleared above). Hence each child of $v$ is in $N_{2}$. Let $u$ be a child of $v$ and $x$ a child of $u$. Let $x$ have a positive weight and assume we could move some weight from $x$ to $u$. If all vertices in the neighborhood of $x$ would remain sufficiently dominated, this would be in contradiction with minimality of the weights in $B(x)$. Hence, no weight can be moved closer to $v$. The algorithm puts $v$ in $D_{1}$ and so this case is completed. 
Suppose $v$ is assigned 2 by one of the two rules. Then simple calculation shows that the new weight of $D \cap B(v)$ is not larger than the old one. Hence it is optimal, and, in addition $v$ has weight 2 , which is best possible.

To see that adding the last vertex to $D$ also yields an optimal W2DF is similar as above and left to the reader. This proves the claim.

If the root $r$ is also put in $D$ then by the above claim, since $B(r)=T$, we derive that $D$ yields an optimal W2DF of $T$. Otherwise, $r$ must be in $N_{2}$, and every component of $T-r$ is optimally dominated, hence $D$ yields an optimal W2DF of $T$.

Corollary 2.3. 2-Rainbow domination function of an arbitrary tree can be determined in linear time and space.

\section{Concluding Remarks}

Remark 1. The problem of describing the graphs that attain the upper bound in Observation 1.2 seems to be very hard (see [8] where some partial results are obtained). We have found a structural characterization of the trees that attain the upper bound when $k=2$, that is those trees for which $\gamma_{\mathrm{r} 2}=2 \gamma$. Because of the length of the resulting description of this class of trees and of the proof that justifies this characterization we omit it.

Remark 2. Is the following relaxation of Vizing's conjecture true: For any graphs $G$ and $H, \gamma_{\mathrm{r} 2}(G \square H) \geq \gamma(G) \gamma(H)$ ?

Since $2 \gamma(G \square H) \geq \gamma_{\mathrm{r} 2}(G \square H)$ this is stronger than the result of Clark and Suen [5]. A similar relaxation was proposed in [2] for the case of integer domination.

\section{REFERENCES}

1. B. Brešar, On Vizing's conjecture, Discuss. Math. Graph Theory, 21 (2001), 5-11.

2. B. Brešar, M. A. Henning and S. Klavžar, On integer domination in graphs and Vizing-like problems, Taiwanese J. Mathematics, 10 (2006), 1317-1328.

3. B. Brešar, M. A. Henning and D. F. Rall, Paired-domination of Cartesian products of graphs, Utilitas Math., To appear.

4. E. J. Cockayne, S. E. Goodman and S. T. Hedetniemi, A linear algorithm for the domination number of a tree, Inform. Process. Lett., 4 (1975), 41-44.

5. W. E. Clark, and S. Suen, An inequality related to Vizing's conjecture, Electron. J. Combin., 7(1) (2000), Note 4, 3, pp. (electronic). 
6. G. Chartrand and L. Lesniak, Graphs \& Digraphs, 3rd Edition, Chapman \& Hall, London, 1996.

7. O. Favaron and M.A. Henning, Paired domination in claw-free cubic graphs, Graphs and Combinatorics, 20 (2004), 447-456.

8. B. Hartnell and D. F. Rall, Domination in Cartesian products: Vizing's Conjecture, In [11], 163-189.

9. B. Hartnell and D. F. Rall, On dominating the Cartesian product of a graph and $K_{2}$, Discuss. Math. Graph Theory 24 (2004), 389-402.

10. T. W. Haynes, S. T. Hedetniemi and P. J. Slater, Fundamentals of Domination in Graphs, Marcel Dekker, New York, 1998.

11. T. W. Haynes, S. T. Hedetniemi and P. J. Slater (eds.), Domination in Graphs: Advanced Topics, Marcel Dekker, New York, 1998.

12. T. W. Haynes and P. J. Slater, Paired-domination in graphs, Networks, 32 (1998), 199-206.

13. T. W. Haynes and P. J. Slater, Paired-domination and the paired-domatic number, Congr. Numer., 109 (1995), 65-72.

14. T. W. Haynes and M. A. Henning, Trees with large paired-domination number, Utilitas Math., To appear.

15. M. A. Henning and D. F. Rall, On the total domination number of Cartesian products of graph, Graphs and Combinatorics, 21 (2005), 63-69.

16. K. E. Proffitt, T. W. Haynes and P. J. Slater, Paired-domination in grid graphs, Congr. Numer, 150 (2001), 161-172.

17. L. Sun, A result on Vizing's conjecture, Discrete Math., 275 (2004), 363-366.

18. V. G. Vizing, Some unsolved problems in graph theory, Uspehi Mat. Nauk, 23(6) (1968), (144), 117-134.

\author{
Boštjan Brešar \\ University of Maribor, \\ FEECS, Smetanova 17, \\ 2000 Maribor, \\ Slovenia \\ E-mail: bostjan.bresar@uni-mb.si \\ Michael A. Henning \\ School of Mathematical Sciences, \\ University of KwaZulu-Natal, \\ Pietermaritzburg Campus, \\ South Africa \\ E-mail: henning@ukzn.ac.za
}


Douglas F. Rall

Department of Mathemtics,

Furman University,

Greenville, SC 29613,

U.S.A.

E-mail: drall@herky.furman.edu 